\title{
E-740 PROPORTIONAL DRIFT TUBE TESTS
}

C. Brown, D. Green, H. Haggerty, S. Hansen, D. Hedin, H. Jostlein, K. Krempetz, S. Kunori, P. Martin, D. Owen, P. Rapp, and M. Takasaki 
E-740 Proportional Drift Tube Tests

C. Brown, D. Green, H. Haggerty, S. Hansen,

D. Hedin, H. Jostlein, K. Krempetz, S. Kunori,

P. Martin, D. Owen, P. Rapp, M. Takasaki

March 15, 1984

I. Design Criteria

The $D \phi$ muon detector ${ }^{1}$ will consist of large area arrays of proportional drift tubes (PDT'S). The beam crossing repetition rate together with the trigger processing time will constrain the maximum drift time to be about one microsecond, corresponding to a drift distance of about $5.0 \mathrm{~cm}$. With such a narrow cell width, it is possible to use rather long cells (up to 6.0 meters) and still keep cell occupancy acceptable. To simplify track reconstruction, space points will be recorded for each track. This will be accomplished using shaped cathode pads to measure the longitudinal coordinate by the ratio of induced charges ${ }^{2}$. 
In order to have minimal thickness of the PDT arrays, a rectangular cross section has been chosen with an aspect ratio of about $3: 1$. This choice has necessitated the use of field shaping electrodes to maintain approximately uniform drift velocities. The test cell presented here incorporates cathode pads which have been designed for both longitudinal position measurement and field shaping.

II. Prototype cell

The prototype test cell is $6.0 \mathrm{~m}$ long with a sensitive cross section $3.8 \mathrm{~cm}$. thick by $10.0 \mathrm{~cm}$ wide. The tube was assembled from interlocking aluminum extrusions (Fig. la). A gap was left between the cathode pad plane and the grounded extrusion wall to reduce the capacitance of the pads to ground. The pads were etched from copper clad G-10 as shown in Fig. 1b. Two sets of charge dividing patterns were used. One set had a repetitive $20.0 \mathrm{~cm}$ pattern (vernier pads) which allowed a precise position measurement (modulo the repetition length). The opposite cathode pad was a single long pattern which determined the longitudinal position with enough precision to locate the correct vernier pad. 
The cell was filled with a mixture of 508 Argon and $50 \%$ ethane. High voltage of $+4.5 \mathrm{kV}$ was applied to the anode wire (50.0 micron gold plated tungsten) and $+1.6 \mathrm{kV}$ to the cathode pads. The aluminum extrusions were at ground potential. The equipotential contours and the contours of equal drift time ${ }^{3}$ are shown in Fig. $2 a$ and Fig. $2 b$.

III. Cosmic Ray Test Setup

A cosmic ray trigger was provided by a pair of scintillators $35.0 \mathrm{~cm}$ by $66.0 \mathrm{~cm}$. A layer of lead, $5.0 \mathrm{~cm}$ thick, was inserted above the bottom scintillator in order to reduce the number of very low energy triggers. The incident cosmic ray position was measured by planes of $2.54 \mathrm{~cm}$ square drift tubes (Fig. 3a). The overall fast electronics scheme is shown in Fig. 3b. The amplifier for the signal on the wire is shown in Fig. 4a. This signal was used to generate a gate for the pad signals, and to provide a drift time measurement for the transverse coordinate. The pad signals were amplified by the circuit shown in Fig. $4 b^{4}$. The sensitivity of this amplifier is $18 \mathrm{mv} / \mathrm{pC}$. With a 500 ns gate into the $A D C$, the net system charge amplification is 180. The intrinsic amplifier noise (assuming about $500 \mathrm{pF}$ chamber capacitance) is $0.4 \mathrm{fC}$. 
IV. Test Results

Figs. $5 a$ and $5 b$ show the difference of the induced charges normalized to the sum of the charges of the two pad pairs. The long pads locate the track to $13.0 \mathrm{~cm}$ (fwhm), which is sufficient to locate the vernier pad. The vernier pads then determine the longitudinal track coordinate to $3.0 \mathrm{~mm}$ (fwhm). This uncertainty in the longitudinal coordinate is primarily caused by electrical pick-up of noise from sources external to the amplifiers. The intrinsic amplifier noise, $0.4 \mathrm{fC}$, is negligible compared to the average pad signal of about $1.0 \mathrm{pC}$. The transverse drift time is shown in Fig. 6. The resolution here $11.5 \mathrm{~mm}$ fwhm) is due to multiple scattering of the incident cosmic ray and alignment uncertainty of the apparatus.

V. Conclusions

The technique of using cathode pad readout for the longitudinal coordinate of a single wire proportional drift tube appears to meet the design requirements of the muon detector at $D \phi$. For the longest tubes, $(6.0 \mathrm{~m})$ some staggering of pads will be necessary to reduce the region of ambiguity near the edges of the short pads. Alternative 
fabrication techniques, including conventional sandwich construction and "snap together" closed aluminum extrusions, are under evaluation.

Acknowledgements

The authors acknowledge the unstinting efforts of C. Lindenmeyer, whose ingenious designs and suggestions are indispensable in this development. 


\section{References}

1. See DǾ Design Report.

2. J. Allison et al., "Diamond shaped cathode pads for the longitudinal coordinate from a drift chamber"; U. of Manchester (preprint)

3. A. Peisert, F. Sauli, Drift and Diffusion of Electrons in Gases: a compilation. CERN 84-08.

4. Prototype $D \emptyset$ calorimeter preamp borrowed from P. Franzini 


\section{Figure Captions}

1. (a) Interlocking aluminum extrusion cross section. (b) Cathode pad layout.

2. (a) Equipotentials calculated for the test cell.

(b) Contours of equal drift time calculated for the test cell filled with 508 Argon, $50 \%$ Carbon dioxide.

3. (a) Cosmic ray trigger apparatus. (b) Fast electronics.

4. (a) Signal wire amplifier. (b) Pad amplifier.

5. (a) Long pad charge ratio normalized to sum of long pad charges. (b) Short pad charge ratio normalized t.o sum of short pad charges.

6. Drift time versus position 


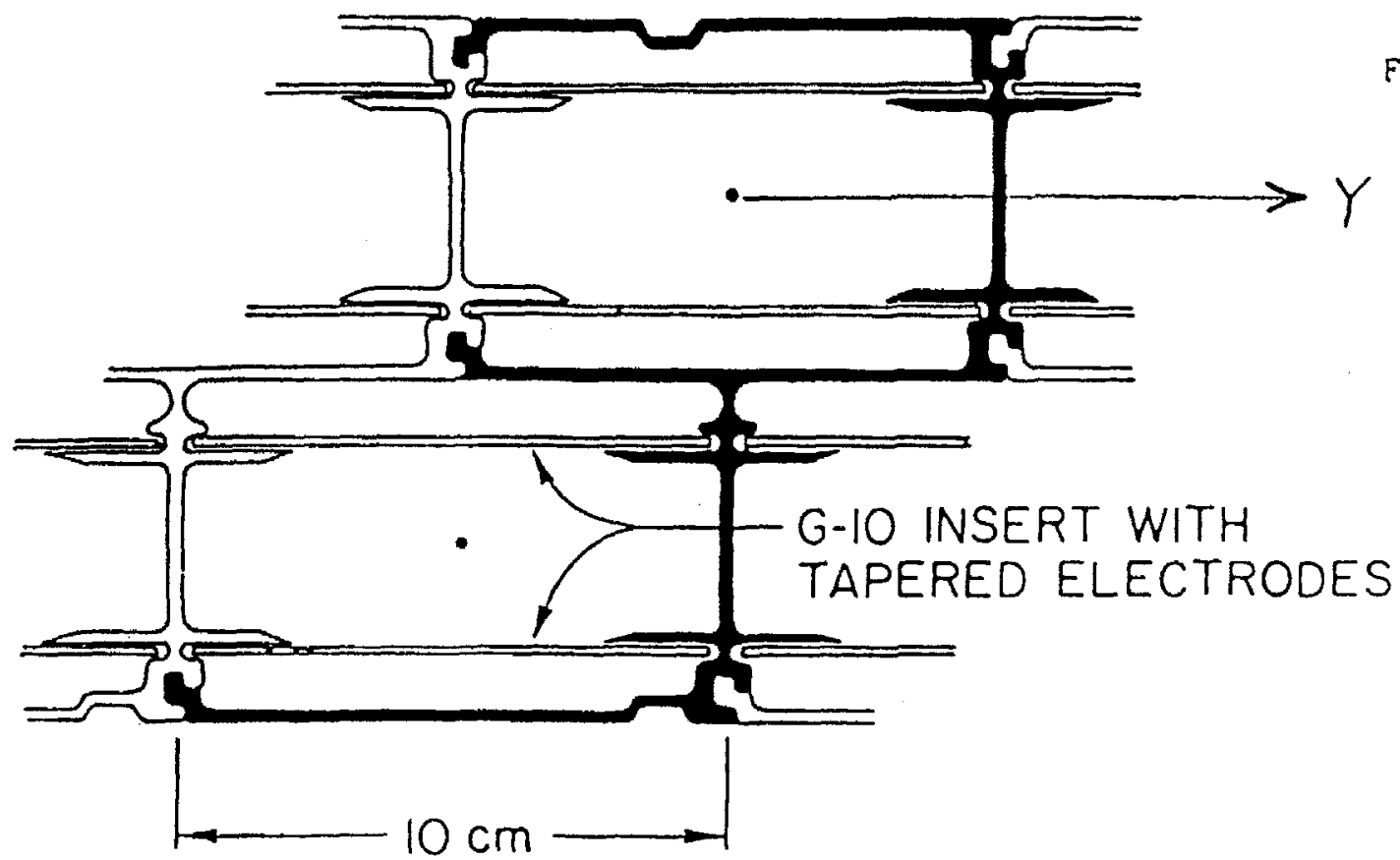

Fig. 1 a.

Fig. Ib.
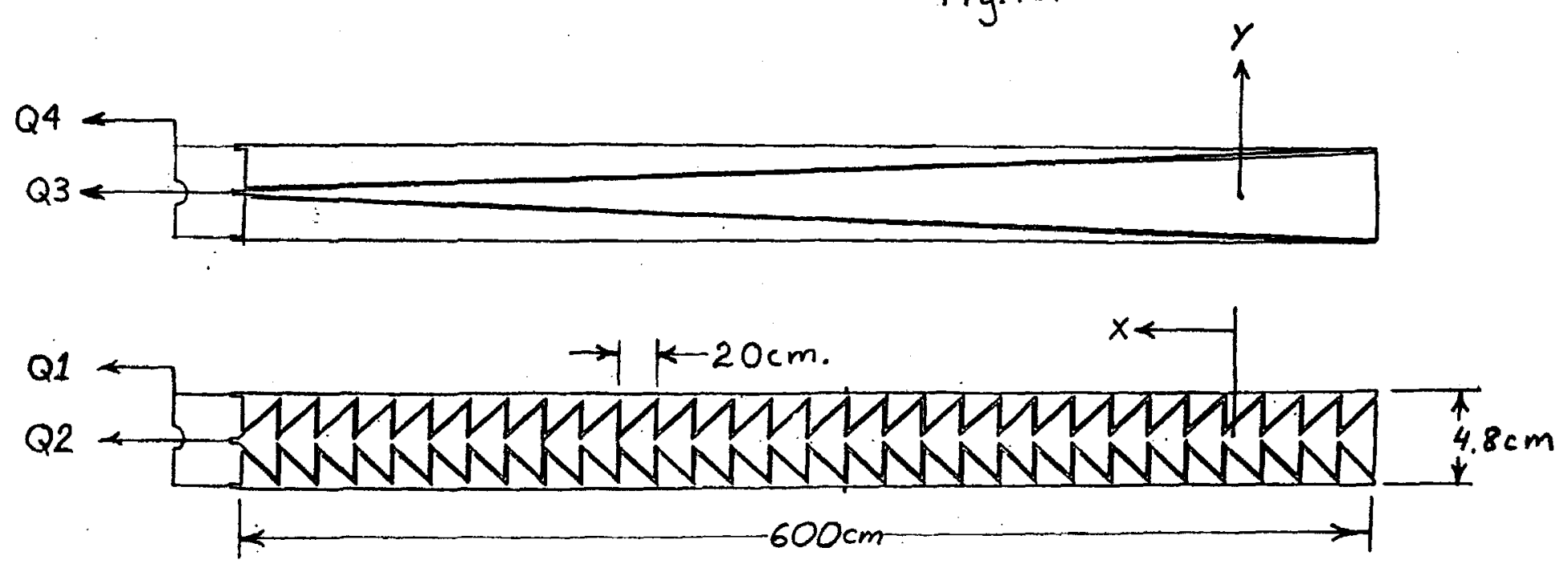
Fig. 2 a

Equipotential Contours
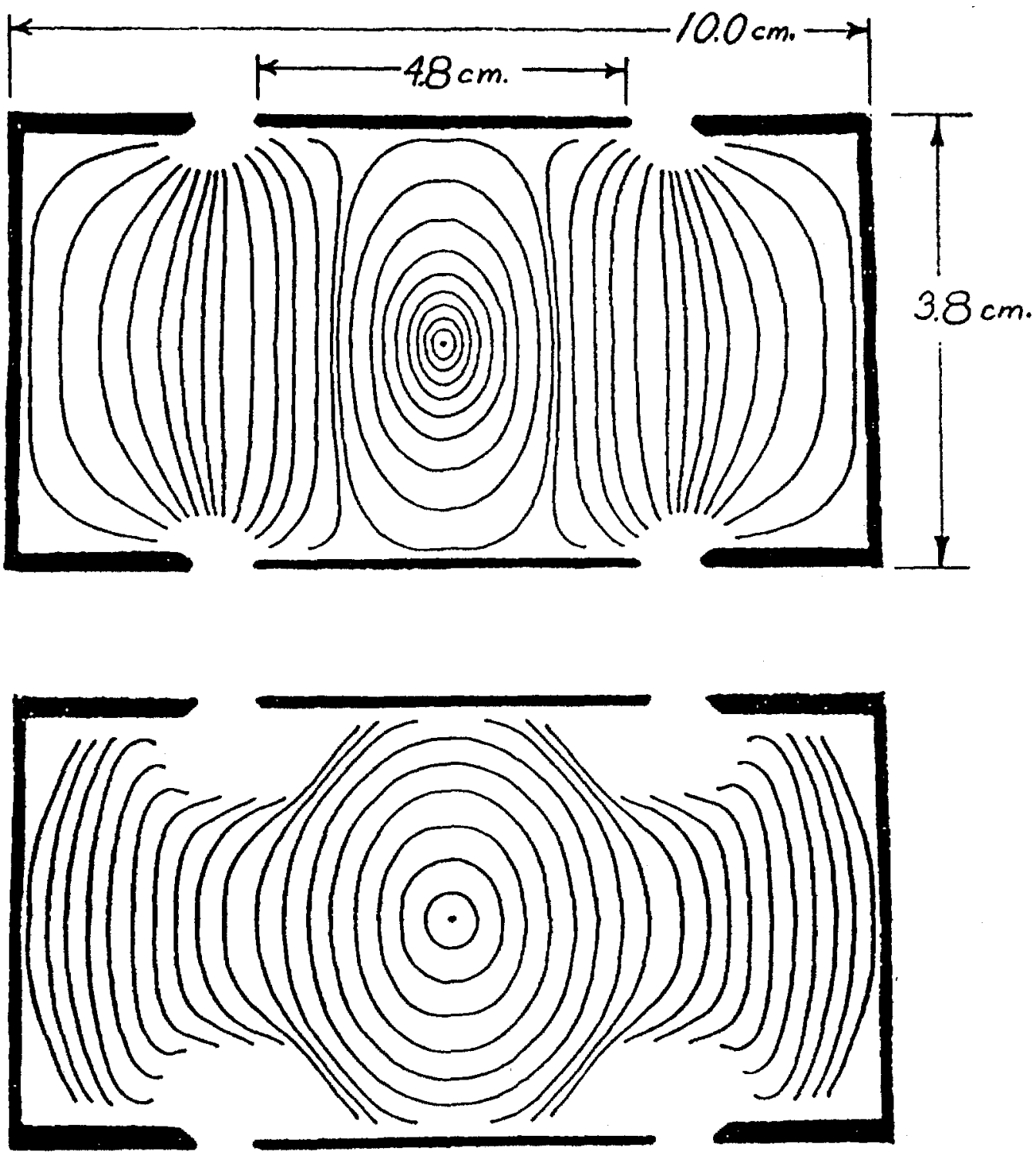

Fig. $2 b$

Equal Drift Time Contours 
Fig. 3a.
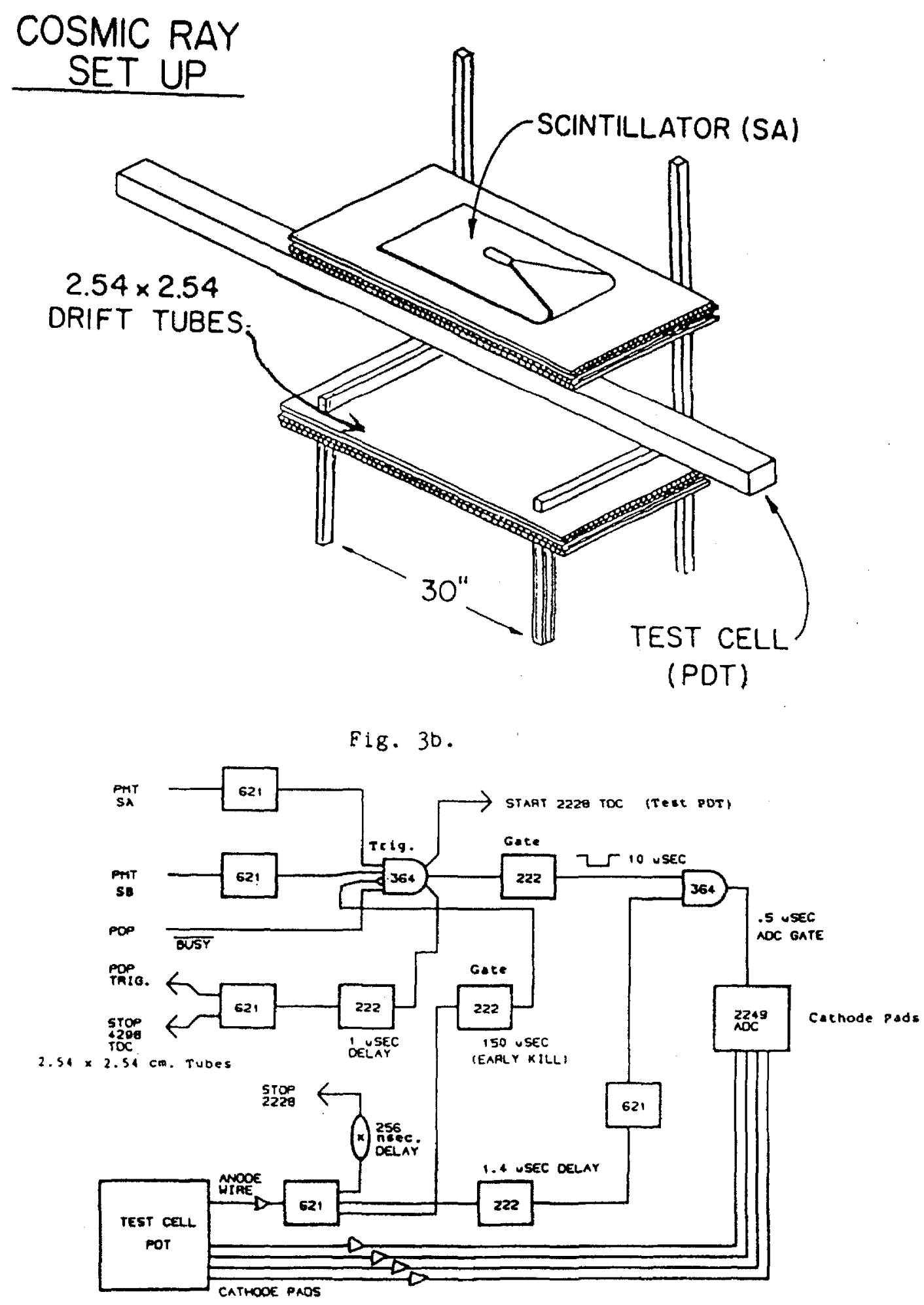

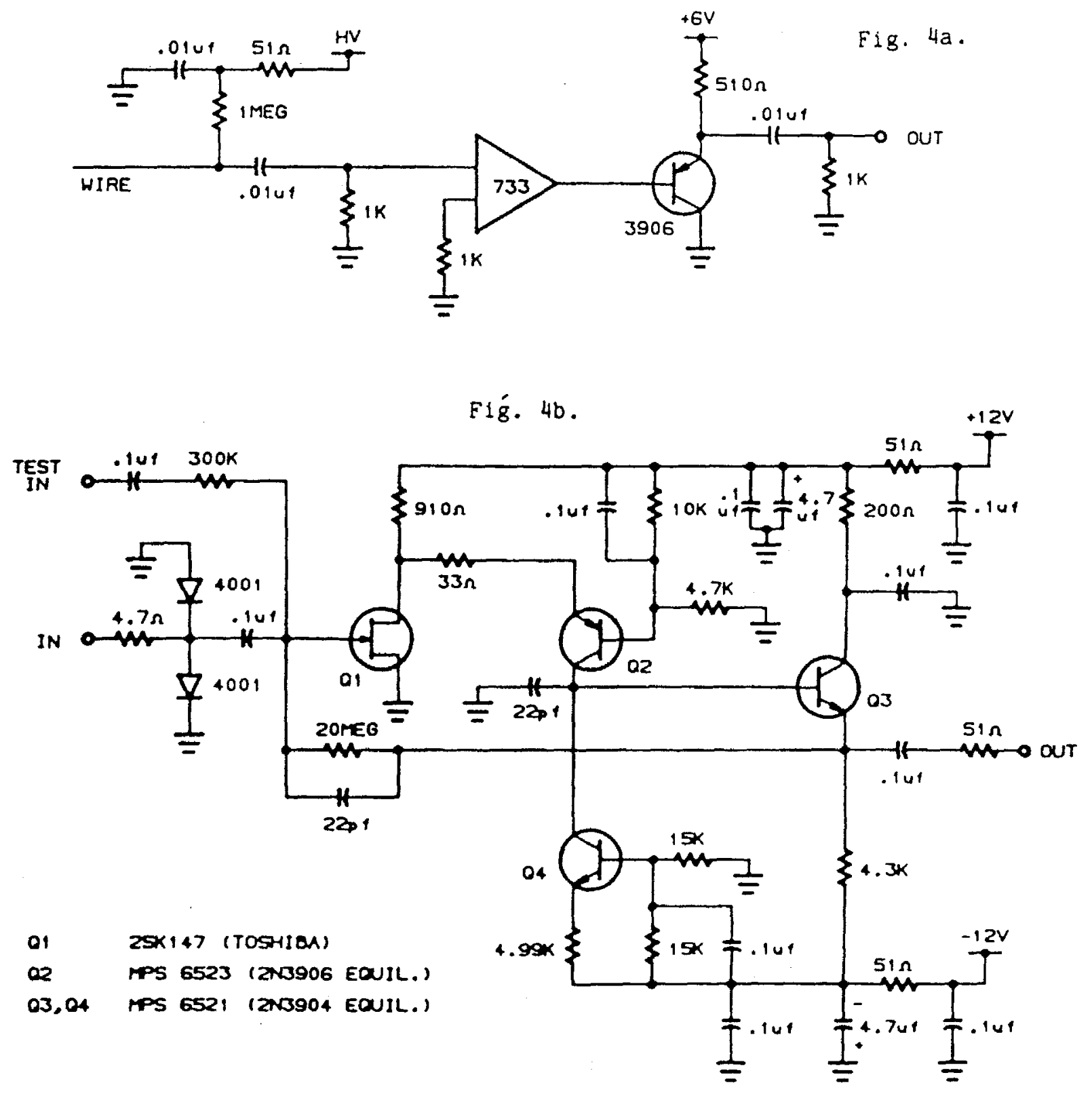
FIG. 52 .

$-12-$
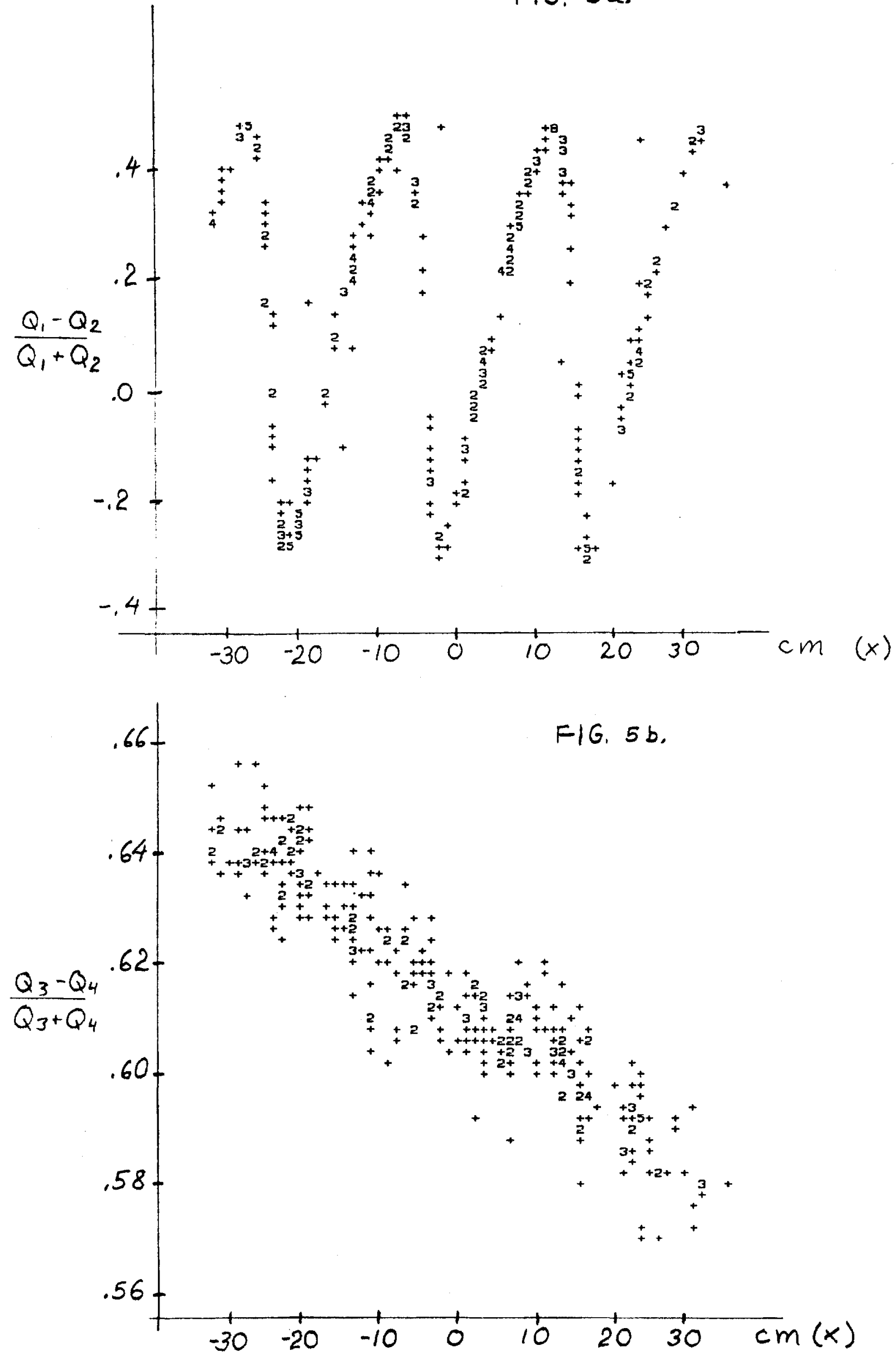
$F \mid G, 6$.

$-13-$

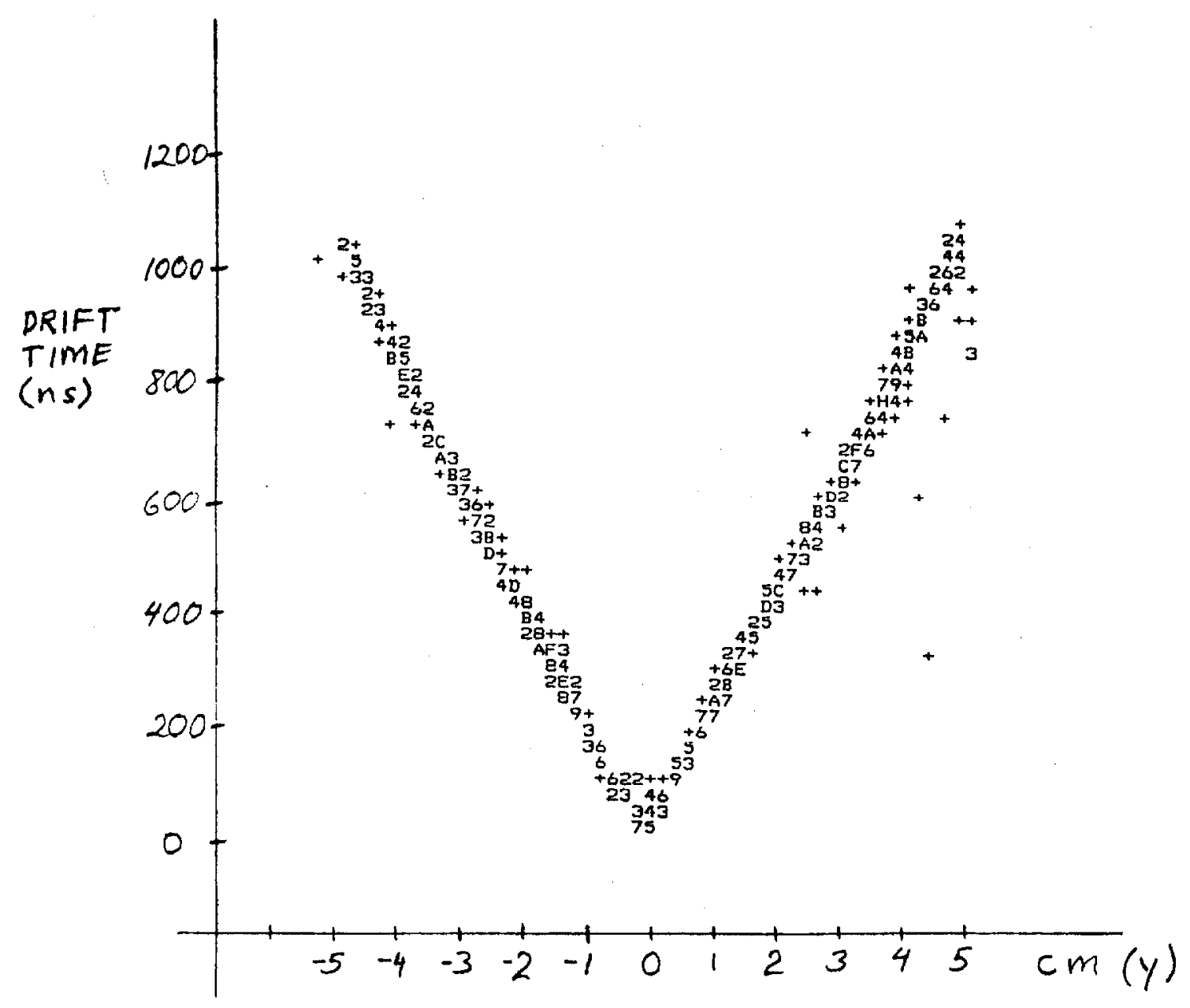

